\title{
Tits rigidity of CAT(0) group boundaries
}

\author{
KHEK LUN HAROLD CHAO \\ ERIC SWENSON
}

\begin{abstract}
We define Tits rigidity for visual boundaries of CAT(0) groups, and prove that the join of two Cantor sets and its suspension are Tits rigid.
\end{abstract}

53C23; 20F67, 51F99

\section{Introduction}

A CAT(0) space $X$ has two natural boundaries with the same underlying point set: the visual boundary $\partial X$ and the Tits boundary $\partial_{T} X$. The obvious bijection from $\partial_{T} X$ to $\partial X$ is continuous, but need not be a homeomorphism.

In the classical case where $X$ is a Riemannian $(n+1)$-manifold of nonpositive sectional curvature, then $\partial X=S^{n}$, so the visual boundary contains very little information (only the dimension). The Tits boundary, on the other hand, is much more interesting. For example, the Tits boundary of $\mathbb{E}^{n+1}$ is also $S^{n}$, while the Tits boundary of $\mathbb{H}^{n+1}$ is discrete. These are of course different for $n>0$. Even in the case where $n=2$, there are at least two other possible Tits boundaries: the Tits boundary of $\mathbb{H}^{2} \times \mathbb{R}$ is the spherical suspension of an uncountable discrete set; and the examples of Croke and Kleiner give the infamous Eye of Sauron pattern. In this paper we examine the other extreme, where the visual topology dictates the Tits metric.

Suppose that $X$ admits a geometric group action. Ruane showed in [11] that if $\partial X$ is a suspension of Cantor set, then $\partial_{T} X$ is the spherical suspension of an uncountable discrete set. In [4] it is shown that if $\partial X$ is the join of two Cantor sets, and if $X$ admits a geometric action by a group $G$ that contains $\mathbb{Z}^{2}$, then $\partial_{T} X$ is isometric to the spherical join of two uncountable discrete sets.

We prove that the same result holds without the $\mathbb{Z}^{2}$ assumption on $G$. We use the action of ultrafilters over $G$ on $\partial X$, whose properties were investigated in [6]. We will also show that if $\partial X$ is the suspension of a join of two Cantor sets, then $\partial_{T} X$ is also the spherical suspension of the spherical join of two uncountable discrete sets. These results suggest the definition of Tits rigidity, and the above results can be rephrased 
by saying that the suspension of a Cantor set, the join of two Cantor sets and the suspension of the join of two Cantor sets are all Tits rigid. On the other hand, a sphere of dimension $n>0$ is not Tits rigid since, as we saw, $\mathbb{E}^{n+1}$ and $\mathbb{H}^{n+1}$ have different Tits boundaries.

The organization of this paper is as follows. This section reviews some basic notions and defines Tits rigidity; Section 2 completes the proof that the join of two Cantor sets is Tits rigid; and Section 3 proves that the suspension of the join of two Cantor sets is Tits rigid. We state some questions in Section 4.

We refer the reader to Bridson and Haefliger [2] or Ballmann [1] for more details.

Definition 1.1 For a metric space $X$ and an interval $I$ of $\mathbb{R}$, an isometric embedding $\alpha: I \rightarrow X$ is called a geodesic. By abuse of notation we will also refer to the image of $\alpha$ as a geodesic.

Definition 1.2 For a geodesic metric space $X$ and a geodesic triangle $\Delta(a, b, c)$ in $X$ with vertices $a, b, c \in X$, there is a Euclidean comparison triangle $\bar{\Delta}=\Delta(\bar{a}, \bar{b}, \bar{c}) \subset \mathbb{E}^{2}$ with $d(a, b)=d(\bar{a}, \bar{b}), d(a, c)=d(\bar{a}, \bar{c})$ and $d(b, c)=d(\bar{b}, \bar{c})$. We define the comparison angle by $\bar{Z}_{a}(b, c)=\angle_{\bar{a}}(\bar{b}, \bar{c})$.

Each point $z \in \Delta(a, b, c)$ has a unique comparison point $\bar{z} \in \bar{\Delta}$. We say that the triangle $\Delta(a, b, c)$ is $\operatorname{CAT}(0)$ if for any $y, z \in \Delta(a, b, c)$ with comparison points $\bar{y}, \bar{z} \in \bar{\Delta}$, we have $d(y, z) \leq d(\bar{y}, \bar{z})$. The space $X$ is said to be CAT(0) if every geodesic triangle in $X$ is $\operatorname{CAT}(0)$.

If $X$ is $\mathrm{CAT}(0)$, then for any geodesic $\alpha:[0, r] \rightarrow X$ and $\beta:[0, s] \rightarrow X$ with $\alpha(0)=$ $\beta(0)=a$, the function

$$
\theta(r, s)=\bar{Z}_{a}(\alpha(r), \beta(s))
$$

is an increasing function of $r, s$. Thus $\lim _{r, s \rightarrow 0} \theta(r, s)$ exists and we call this limit $\angle_{a}(\alpha(r), \beta(s))$. It follows that for any $a, b, c$ in a $\mathrm{CAT}(0)$ space,

$$
\angle_{a}(b, c) \leq \bar{Z}_{a}(b, c) \text {. }
$$

Recall that a metric space is proper if closed metric balls are compact. Recall also that an action by isometries of a group $G$ on a space $X$ is geometric if the action is properly discontinuous and cocompact.

In this paper, $G$ will be a group and $X$ an unbounded proper CAT(0) space on which $G$ acts geometrically.

The (visual) boundary $\partial X$ is the set of equivalence classes of rays, where rays are equivalent if they are within finite Hausdorff distance of each other. Given a ray $R$ and 
a point $x \in X$, there is a ray $S$ emanating from $x$ with $R \sim S$. Fixing a base point $\mathbf{0} \in X$, we define the visual topology on $\bar{X}=X \cup \partial X$ by taking the basic open sets about $x \in X$ to be the open metric balls about $x$. For $y \in \partial X$ and a ray $R$ from $\mathbf{0}$ representing $y$, we construct basic open sets $U(R, n, \epsilon)$, where $n, \epsilon>0$. We say that $z \in U(R, n, \epsilon)$ if the unit speed geodesic $S:[0, d(\mathbf{0}, z)] \rightarrow \bar{X}$ from $\mathbf{0}$ to $z$ satisfies $d(R(n), S(n))<\epsilon$. These sets form a basis for a regular topology on $\bar{X}$ and $\partial X$. For any $x \in X$ and $u, v \in \partial X$, we can define $L_{x}(u, v)$ and $\bar{L}_{x}(u, v)$ by parametrizing the rays $[x, u)$ and $[x, v)$ by $t \in[0, \infty)$ and taking the limit of $\bar{L}_{x}$ as $t \rightarrow 0$ and as $t \rightarrow \infty$ respectively.

For $u, v \in \partial X$, we define $\angle(u, v)=\sup _{p \in X} \angle_{p}(u, v)$. It follows from Bridson and Haefliger [2, II 9.8] that $\angle(u, v)=\bar{L}_{p}(u, v)$ for any $p \in X$. Notice that isometries of $X$ preserve the angle between points of $\partial X$. This defines a metric called the angle metric on the set $\partial X$. The angle metric defines a path metric $d_{T}$ on the set $\partial X$, called the Tits metric, whose topology is at least as fine as the visual topology of $\partial X$. Also $\angle(a, b)$ and $d_{T}(a, b)$ are equal whenever either of them is less than $\pi$. For any $u \in \partial X$, we define $B_{T}(u, \epsilon)=\left\{v \in \partial X: d_{T}(u, v)<\epsilon\right\}$ and $\bar{B}_{T}(u, \epsilon)=\left\{v \in \partial X: d_{T}(u, v) \leq \epsilon\right\}$.

The set $\partial X$ with the Tits metric is called the Tits boundary of $X$, denoted $\partial_{T} X$. Isometries of $X$ extend to isometries of $\partial_{T} X$.

The identity function $\partial_{T} X \rightarrow \partial X$ is continuous, but the identity function $\partial X \rightarrow \partial_{T} X$ is only lower semi-continuous. That is, for any sequences $\left(u_{n}\right),\left(v_{n}\right) \subset \partial X$ with $u_{n} \rightarrow u$ and $v_{n} \rightarrow v$ in $\partial X$, we have

$$
\underline{\lim } d_{T}\left(u_{n}, v_{n}\right) \geq d_{T}(u, v) .
$$

Definition 1.3 A subgroup $H<G$ is called convex if there exists a closed convex $A \subset X$ with $H$ acting geometrically on $A$.

Definition 1.4 For $g \in G$, we define $\tau(g)=\inf _{x \in X} d(x, g(x))$. This minimum is realized, and $\operatorname{Min}(g)=\{x \in X: d(x, g(x))=\tau(g)\}$ is nonempty.

For any $g \in G$, the centralizer $Z_{g}$ is a convex subgroup that acts geometrically on $\operatorname{Min}(g)$, which is closed and convex by Ruane [10, 3.2], and Bridson and Haefliger [2, II 6.8]. In fact, if $g$ is hyperbolic, then $\operatorname{Min}(g)=A \times Y$, where $Y$ is a closed convex subset of $X$ on which $Z_{g} /\langle g\rangle$ acts geometrically (see [2, II 6.2] and [12, Lemma 14]), and $A$ is an axis of $g$.

Definition 1.5 The boundary of a CAT( 0$)$ space will be called a CAT( 0$)$ boundary. If $G$ is a group acting geometrically on a $\operatorname{CAT}(0)$ space $X$, then $\partial X$ is called a CAT( 0$)$ 
boundary of $G$, or we say $\partial X$ is a $\operatorname{CAT}(0)$ group boundary. In all cases, a CAT( 0$)$ boundary comes equipped with both the visual topology and the Tits metric (which normally gives a finer topology).

Definition 1.6 Let $A$ and $B$ be boundaries of CAT(0) spaces. A function $f: A \rightarrow B$ is called a boundary isomorphism if $f$ is a homeomorphism in the visual topology and $f$ is an isometry in the Tits metric. A function $g: A \rightarrow B$ is called a boundary embedding if $g$ is a boundary isomorphism onto its image, where the metric on $g(A)$ is the restriction of the Tits metric.

Two boundaries of the same CAT(0) group need not be boundary-isomorphic to each other, or even homeomorphic to each other; see Croke and Kleiner [5].

Definition 1.7 For $A \subset X, \Lambda A$ is the set of limit points of $A$ in $\partial X$. For $H<G$, we define $\Lambda H=\Lambda(H x)$, where $H x$ is the $H$-orbit of some $x \in X$ (this is independent of the choice of $x$ ).

Lemma 1.8 Let $Y$ be a closed, convex subset of $X$. Inclusion of $Y$ into $X$ induces a topological embedding $\iota: \partial Y \rightarrow \Lambda Y$ of $\partial Y$ in $\partial X$. Also, $\iota$ is isometric for the angle metric. Furthermore, if diam $\partial_{T} Y \leq \pi$, then $\iota: \partial_{T} Y \rightarrow \partial_{T} X$ is a boundary embedding.

Proof Since the inclusion $Y \rightarrow X$ is isometric, geodesics in $Y$ are geodesics in $X$, so by choosing a base point $y \in Y$ we have $\partial Y \subset \partial X$, which defines $\iota$. Also, for a geodesic $R$ in $Y$ from $y, U_{X}(R, n, \epsilon) \cap Y=U_{Y}(R, n, \epsilon)$, where $U_{X}$ is the neighborhood in $X$ and $U_{Y}$ the neighborhood in $Y$. Thus $\iota: \partial Y \rightarrow \partial X$ is an embedding with image $\Lambda Y$. Since $Y$ is isometrically embedded in $X$, for any $\alpha, \beta \in \Lambda Y, \bar{Z}_{y}(\alpha, \beta)$ is the same in both $X$ and $Y$. It follows that $\iota$ is isometric for the angle metric, and so $\iota: \partial_{T} Y \rightarrow \partial_{T} X$ will be Lipschitz 1.

Now suppose diam $\partial_{T} Y \leq \pi$. This implies that the set $S=\{(\alpha, \beta) \in \partial Y \times \partial Y$ : $\left.d_{T}(\alpha, \beta)<\pi\right\}$ is dense in $\partial_{T} Y \times \partial_{T} Y$. Since the angle metric and the Tits metric are the same when either is less than $\pi, d_{T} \circ(\iota \times \iota)=d_{T}$ on $S$, and it follows that $\iota: \partial_{T} Y \rightarrow \partial_{T} X$ is an isometric embedding.

A line in the Euclidean plane gives an example where $\iota$ is not a boundary embedding.

Definition 1.9 A compact metrizable space $Y$ is said to be Tits rigid if for any two CAT(0) group boundaries $Z_{1}$ and $Z_{2}$ homeomorphic to $Y, Z_{1}$ is boundary isomorphic to $Z_{2}$. 
Definition 1.10 (Bridson and Haefliger [2]) For topological spaces $Y_{1}, Y_{2}$, we define the topological join $Y_{1} * Y_{2}$ to be the quotient

$$
Y_{1} \times Y_{2} \times\left[0, \frac{\pi}{2}\right] /\left\{\begin{array}{cl}
\left(a, b_{1}, 0\right) \sim\left(a, b_{2}, 0\right) & \text { for } a \in Y_{1}, b_{1}, b_{2} \in Y_{2} \\
\left(a_{1}, b, \frac{\pi}{2}\right) \sim\left(a_{2}, b, \frac{\pi}{2}\right) & \text { for } a_{1}, a_{2} \in Y_{1}, b \in Y_{2}
\end{array}\right\} .
$$

We will refer to $Y_{1} \times Y_{2} \times\{0\}$ as $Y_{1}$ and we will refer to $Y_{1} \times Y_{2} \times\left\{\frac{\pi}{2}\right\}$ as $Y_{2}$. For fixed $y_{i} \in Y_{i}$, the arc $\left(y_{1}, y_{2}, t\right), t \in\left[0, \frac{\pi}{2}\right]$ will be called the join arc from $y_{1}$ to $y_{2}$.

For metric spaces $Y_{1}$ and $Y_{2}$ with metrics bounded by $\pi$, the spherical join $Y_{1} *_{S} Y_{2}$ is the point set $Y_{1} * Y_{2}$ endowed with the metric

$$
\begin{aligned}
d\left(\left(y_{1}, y_{2}, \theta\right),\left(y_{1}^{\prime},\right.\right. & \left.\left.y_{2}^{\prime}, \theta^{\prime}\right)\right) \\
& =\arccos \left[\cos \theta \cos \theta^{\prime} \cos \left(d\left(y_{1}, y_{1}^{\prime}\right)\right)+\sin \theta \sin \theta^{\prime} \cos \left(d\left(y_{2}, y_{2}^{\prime}\right)\right)\right] .
\end{aligned}
$$

For a topological space $Y$, we define the suspension $\Sigma Y$ to be the topological join of $Y$ with a discrete two-point set $\{n, p\}$. In this setting we refer to the join arcs as suspension arcs. For a metric space $Y$ with metric bounded by $\pi$, we define the spherical suspension $\Sigma_{S} Y$ to be the spherical join of $Y$ with $\{n, p\}$, where $d(n, p)$ is defined to be $\pi$.

\subsection{Ultrafilters and pulling}

Recall that an ultrafilter $\omega$ on $G$ is a function $\omega: 2^{G} \rightarrow\{0,1\}$ that satisfies these conditions:

- $\omega(G)=1$.

- $\omega(\varnothing)=0$.

- $\omega(A \cup B)=\omega(A)+\omega(B)-\omega(A \cap B)$.

It follows that, for any $A \subset G$, either $\omega(A)=1$ or $\omega(G-A)=1$, but not both. For $a \in G$, the principal ultrafilter $\delta_{a}$ is defined as

$$
\delta_{a}(B)= \begin{cases}1 & \text { if } a \in B \\ 0 & \text { if } a \notin B .\end{cases}
$$

It follows that $\omega$ is principal if and only if $\omega(F)=1$ for some finite $F \subset G$. If $\omega$ is not principal we call it a nonprincipal ultrafilter, or NPUF. By the axiom of choice, there exist NPUFs on $G$. For a NPUF $\omega$ on $G, \omega(F)=0$ for all finite $F \subset G$. For any infinite $A \subset G$, there exists a NPUF $\omega$ on $G$ with $\omega(A)=1$.

For an ultrafilter $\omega$ on $G$ and $y \in \partial X$, we define the ultralimit of $y$ with respect to $\omega$ to be $\lim _{g \rightarrow \omega} g(y)=z \in \partial X$ if for any neighborhood $U$ of $z$ in $\partial X$,

$$
\omega\{g \in G: g(y) \in U\}=1 .
$$


It turns out that the ultralimit always exists and is unique. We will denote $\lim _{g \rightarrow \omega} g(y)$ by $T^{\omega}(y)$, and think of $T^{\omega}$ as a (generically horribly discontinuous) function from $\partial X$ to $\partial X$, or if we are feeling more charitable we will think of $T^{\omega}: \partial_{T} X \rightarrow \partial_{T} X$ as a Lipschitz-1 map (see [6, Section 1.1] for more details).

For a NPUF $\omega$ on $G$, we say that $\omega$ pulls from $n \in \partial X$ if there is a geodesic ray $S \subset X$ representing $n$ and a compact set $C \subset X$ with $\omega\{g \in G: g(S) \cap C \neq \varnothing\}=1$. We now translate [12, Lemma 7] into the language of ultrafilters:

Lemma 1.11 If $\omega$ is a NPUF of $G$ pulling from $n \in \partial X$, there is a point $p \in \partial X$ with $d_{T}\left(p, T^{\omega} n\right)=\pi$ such that $T^{\omega}$ maps $\partial_{T} X$ into the set of Tits geodesics of length $\pi$ from $T^{\omega} n$ to $p$. Furthermore, for any $a \in \bar{B}_{T}(n, \pi), T^{\omega}$ is an isometry on a Tits geodesic $[n, a]$ from $n$ to $a$.

Acknowledgements This work was partially supported by grant number 209403 from the Simons Foundation.

\section{The join of two Cantor sets is Tits rigid}

Suppose that $\partial X$ is topologically the suspension of two Cantor sets $C_{1}$ and $C_{2}$, so $\partial X \cong C_{1} * C_{2}$. Replacing $G$ with a subgroup of index at most 2 , we may assume that $C_{1}$ and $C_{2}$ are $G$-invariant. By [4, Lemma 2.3] the action of $G$ on $X$ is not of rank 1. Thus, by [9], since $\partial X$ is one-dimensional, $\operatorname{diam}\left(\partial_{T} X\right) \leq 4 \pi / 3$.

If $g \in G$ with $\left\{g^{ \pm}\right\} \not \subset C_{i}$ for $i=1,2$, then we are done by [4, Lemma 3.7 and the discussion above it], since if $\partial \operatorname{Min}(g)$ is the boundary of a flat, then $Z_{g}$ is virtually $\mathbb{Z}^{2}$ and the result follows from [4]. Thus we may assume that there are infinitely many hyperbolic elements $g \in G$ with $\left\{g^{ \pm}\right\} \subset C_{1}$. Let $\alpha=d_{T}\left(C_{1}, C_{2}\right)$.

By compactness, there will be points of $C_{1}$ and $C_{2}$ realizing this minimum. Since $C_{1}$ and $C_{2}$ are closed invariant subsets of $\partial X, d\left(p, C_{i}\right) \leq \pi / 2$ for any $p \in \partial X$ and $i=1,2$ by [9, Theorem 23], so $\alpha \leq \pi / 2$. For $a \in C_{i}$ and $b \in C_{3-i}$, let $\overline{a b}$ be the suspension arc from $a$ to $b$. For any path $\gamma$ in $\partial(X)$, let $\ell(\gamma)$ be the Tits length of the path $\gamma$ (which may be $\infty$ ).

Lemma 2.1 Fix $1 \leq i \leq 2$. Let $a \in C_{i}$ and $b \in C_{3-i}$. There exists $c \in C_{3-i}-\{b\}$ such that $\ell(\overline{a b})+\ell(\overline{a c}) \leq \pi$.

Proof Suppose not. Then for all $c \in C_{3-i}-\{b\}, \ell(\overline{a b})+\ell(\overline{a c})>\pi$. 
First consider the case where $\ell(\overline{a b})>\pi / 2$. By lower semi-continuity, $d_{T}\left(a, C_{3-i}\right)+$ $\ell(\overline{a b})>\pi$. We can choose $p \in \overline{a b}$ with $\ell(\overline{p b})>\pi / 2$ and $\overline{a p}+d_{T}\left(a, C_{3-i}\right)>\pi / 2$. Thus $d\left(p, C_{3-i}\right)>\pi / 2$, a contradiction.

Now consider the case where $\ell(\overline{a b}) \leq \pi / 2$. It follows that $\ell(\overline{a b})=d_{T}\left(a, C_{3-i}\right)$. Thus for any $c \in C_{3-i}-\{b\}$ there is a point $p \in \overline{a c}$ with $\ell(\overline{p c})>\pi / 2$ and $\ell(\overline{a p})+\ell(\overline{a b})>$ $\pi / 2$. It follows that $d_{T}\left(p, C_{3-i}\right)>\pi / 2$, a contradiction.

We then get the following obvious consequence.

Corollary 2.2 For any $a \in C_{1}$ and $b \in C_{2}, \ell(\overline{a b}) \leq \pi-\alpha$.

Lemma 2.3 Fix $1 \leq i \leq 2$ and suppose that $d_{T}\left(b, C_{3-i}\right)>\alpha$ for some $b \in C_{i}$. Then:

(1) $\alpha<\frac{\pi}{4}$.

(2) $d_{T}\left(b, C_{3-i}\right) \leq \frac{\pi}{2}-\alpha$.

(3) $\ell(\overline{b c}) \leq \pi-2 \alpha-d_{T}\left(b, C_{3-i}\right)$ for all $c \in C_{3-i}$.

Proof The subset $A_{i}=\left\{a \in C_{i}: d_{T}\left(a, C_{3-i}\right)=\alpha\right\}$ is closed and $G$-invariant. It follows that

$$
\frac{\pi}{2} \geq d_{T}\left(b, A_{i}\right) \geq d_{T}\left(b, C_{3-i}\right)+d_{T}\left(C_{3-i}, A_{i}\right)=d_{T}\left(b, C_{3-i}\right)+\alpha,
$$

and we have (1) and (2).

Now let $c \in C_{3-i}$. If $\ell(\overline{b c})>\pi-2 \alpha-d_{T}\left(b, C_{3-i}\right)$, then there is a point $p \in \overline{b c}$ with $\ell(\overline{p c})+\alpha>\pi / 2$ and $\ell(\overline{b p})+d_{T}\left(b, C_{3-i}\right)+\alpha>\pi / 2$. It follows that $d_{T}\left(p, A_{i}\right)>\pi / 2$, a contradiction.

Theorem 2.4 Let $\omega$ be an ultrafilter on $G$ and $c_{i} \in C_{i}$ for $i=1,2$. Let $\hat{c}_{i}=T^{\omega}\left(c_{i}\right)$ for $i=1,2$. Then $T^{\omega}\left(\overline{c_{1} c_{2}}\right)=\overline{\hat{c}_{1} \hat{c}_{2}}$.

Proof Let $\pi: C_{1} \times C_{2} \times[0,1] \rightarrow C_{1} * C_{2}$ be the quotient map. Since $C_{i}$ is $G$-invariant for $i=1,2, T^{\omega}\left(C_{i}\right) \subset C_{i}$.

Notice that for each $g \in G, g\left(\overline{c_{1} c_{2}}\right)=\overline{g\left(c_{1}\right) g\left(c_{2}\right)}$. Suppose that for some $b \in\left[c_{1}, c_{2}\right]$, $\hat{b}=T^{\omega}(b) \notin \overline{\hat{c}_{1} \hat{c}_{2}}$. Then there exist open neighborhoods $U_{i}$ of $\hat{c}_{i}$ in $C_{i}$ for $i=1,2$ and an open set $V \ni \hat{b}$ of $C_{1} * C_{2}$ with $\pi\left(U_{1} \times U_{2} \times[0,1]\right) \cap V=\varnothing$. Notice that $\omega\left\{g \in G: g\left(c_{i}\right) \subset U_{i}\right\}=1$ for $i=1,2$. However,

$$
\left\{g \in G: g(b) \in \pi\left(U_{1} \times U_{2} \times[0,1]\right)\right\}=\bigcap_{i=1}^{2}\left\{g \in G: g\left(c_{i}\right) \subset U_{i}\right\},
$$


and so

$$
\omega\left\{g \in G: g(b) \in \pi\left(U_{1} \times U_{2} \times[0,1]\right)\right\}=1 .
$$

We then get $\omega\{g \in G: g(b) \in V\}=0$, which is a contradiction. It follows that $T^{\omega}\left(\overline{c_{1} c_{2}}\right) \subseteq \overline{\hat{c}_{1} \hat{c}_{2}}$. However, by Lemma $2.1, \ell\left(\overline{c_{1} c_{2}}\right) \leq \pi$, so $\overline{c_{1} c_{2}}$ is connected in the Tits metric, and since $T^{\omega}$ is Lipschitz on $\partial_{T} X, T^{\omega}\left(\overline{c_{1} c_{2}}\right)$ is connected, and therefore $T^{\omega}\left(\overline{c_{1} c_{2}}\right)=\overline{\hat{c}_{1} \hat{c}_{2}}$.

Lemma 2.5 Let $g \in G$ be hyperbolic, with $\left\{g^{ \pm}\right\} \subset C_{1}$. If $\alpha<\pi / 2$, then there are infinitely many $c \in C_{2}$ with $\ell\left(\overline{g^{+} c}\right)=d_{T}\left(g^{+}, C_{2}\right)$.

Proof By lower semi-continuity, there exists $c \in C_{2}$ with $\ell\left(\overline{g^{+} c}\right)=d_{T}\left(g^{+}, C_{2}\right)$. If any positive power of $g$ fixes $c$, then by [12, Corollary 10, Lemma 11, Theorem 13] we have $\mathbb{Z}^{2}<G$ and by [4], $\alpha=\pi / 2$. Thus the $\langle g\rangle$-orbit of $c$ is infinite, and all points $b$ in this orbit satisfy

\section{Theorem 2.6}

$$
\begin{gathered}
\ell\left(\overline{g^{+} b}\right)=d_{T}\left(g^{+}, C_{2}\right) . \\
\alpha=\frac{\pi}{2}
\end{gathered}
$$

Proof We assume $\alpha<\pi / 2$.

Case I For any $x \in C_{1}, d_{T}\left(x, C_{2}\right)=\alpha$. By hypothesis, there exists a hyperbolic element $g \in G$ with $g^{ \pm} \in C_{1}$, and we let $b=g^{+}$. Using Lemma 2.5, there exist distinct $c, d \in C_{2}$ with $\ell(\overline{b c})=\alpha=\ell(\overline{b d})$. Choose $a \neq b$, with $a \in C_{1}$, and then choose $e \in C_{2}$ with $\ell(\overline{a e})=\alpha$. By Corollary 2.2, $\ell(\overline{a c}), \ell(\overline{a d}), \ell(\overline{b e}) \leq \pi-\alpha$. Each of the loops $a e b d, a e b c$ and $a d b c$ which is nontrivial must have length at least $2 \pi$. It follows that $\ell(\overline{a c}), \ell(\overline{a d}), \ell(\overline{b e})=\pi-\alpha$. Let $m$ be the midpoint of the segment $\overline{b c}$. Let $\omega$ be a NPUF of $G$ pulling from $m$. Let $T^{\omega}(a)=\hat{a}, T^{\omega}(b)=\hat{b}, T^{\omega}(c)=\hat{c}$, $T^{\omega}(d)=\hat{d}, T^{\omega}(e)=\hat{e}$, and $T^{\omega}(m)=\hat{m}$. By Lemma $1.11, T^{\omega}$ is an isometry on each Tits segment of length at most $\pi$ from $m$ and $T^{\omega}(\partial X)$ is contained in the set of all Tits geodesics of length $\pi$ from $\hat{m}$ to some point $\hat{p} \in \partial X$. Since these geodesics can branch only at $\hat{m}$ and $\hat{p}$, it follows that $T^{\omega}(\overline{b d}) \subset T^{\omega}(\overline{b e})$. However, by Theorem 2.4,

$$
T^{\omega}(\overline{b d})=\overline{\hat{b} \hat{d}} \quad \text { and } \quad T^{\omega}(\overline{b e})=\overline{\hat{b} \hat{e}} .
$$

Thus $\overline{\hat{b} \hat{d}} \subset \overline{\hat{b}} \hat{e}$, and it follows by definition that $\hat{d}=\hat{e}$.

Since $T^{\omega}$ is an isometry on any Tits segment from $m$ of length at most $\pi$ and $d_{T}(c, m)<d_{T}(d, m) \leq \pi, \hat{c} \neq \hat{d}$, and similarly $\hat{a} \neq \hat{b}$. Thus the loop $\hat{a} \hat{c} \hat{b} \hat{d}$ is nontrivial. Since $T^{\omega}$ is Lipschitz with constant one for the Tits metric,

$$
\ell(\overline{\hat{b} \hat{d}})=\alpha=\ell(\overline{\hat{b} \hat{c}}) .
$$



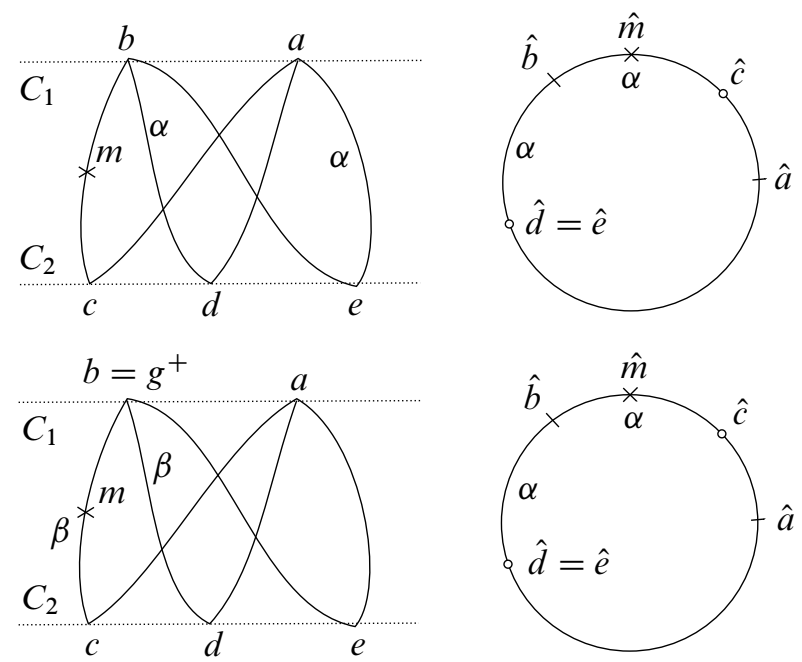

Figure 1: Proof of Theorem 2.6. Top: Case I. Bottom: Case II.

Since $\hat{e}=\hat{d}, \ell(\overline{\hat{a} \hat{d}})=\alpha$, and finally $\ell(\overline{\hat{a} \hat{c}}) \leq \pi-\alpha$. Thus the nontrivial loop $\hat{a} \hat{c} \hat{b} \hat{d}$ has $\ell(\hat{a} \hat{c} \hat{b} \hat{d}) \leq 3 \alpha+\pi-\alpha=\pi+2 \alpha<2 \pi$ since $\alpha<\pi / 2$. This is a contradiction.

Case II There is $x \in C_{1}$ with $d_{T}\left(x, C_{2}\right)>\alpha$. Let $g \in G$ be hyperbolic with $\left\{g^{ \pm}\right\} \subset C_{1}$, and let $b=g^{+}$and $\beta=d_{T}\left(b, C_{2}\right)$. Notice that $\beta<\pi / 2$ by Lemma 2.3. Using Lemma 2.5, there are distinct elements $c, d \in C_{2}$ with

$$
\ell(\overline{b c})=\beta=\ell(\overline{b d}) .
$$

Choose $a \neq b$, with $a \in C_{1}$, and then choose $e \in C_{2}$ with $\ell(\overline{a e})=\alpha$. We now proceed as in Case I, pulling from $m$ the midpoint of $\overline{b c}$. We obtain, as before, a nontrivial loop $\hat{a} \hat{c} \hat{b} \hat{d}$. However, this time we have

$$
\ell(\overline{\hat{b} \hat{d}}), \ell(\overline{\hat{b} \hat{c}}) \leq \beta
$$

Arguing as in Case I, $\ell(\overline{\hat{a} \hat{d}})=\alpha$ and $\ell(\overline{\hat{a} \hat{c}}) \leq \pi-\alpha$. Thus, the nontrivial loop $\hat{a} \hat{c} \hat{b} \hat{d}$ has

$$
\ell(\hat{a} \hat{c} \hat{b} \hat{d}) \leq 2 \beta+\alpha+\pi-\alpha=\pi+2 \beta<2 \pi
$$

since $\beta<\pi / 2$, and we have the same contradiction as before.

Theorem 2.7 The join of two Cantor sets is Tits rigid.

Proof By Theorem 2.6, $\alpha=\pi / 2$, and so by Corollary 2.2, for any $a \in C_{1}$ and $b \in C_{2}$, $\ell(\overline{a b})=\pi / 2$. 
Let $\hat{Z}=C_{1} * S C_{2}$ be the spherical join, where the metric on $C_{1}$ is always $\pi$ for distinct points and similarly for $C_{2}$, so both are discrete as metric spaces. Let $Z=C_{1} * C_{2}$ be the topological join (notice that $C_{1}$ and $C_{2}$ are not discrete here). Notice that $\hat{Z}=Z$ as point sets.

Define $\Phi: \hat{Z} \rightarrow \partial_{T} X$ by $\Phi$ being the identity on $C_{1}$ and $C_{2}$ and $\Phi\left(c_{1}, c_{2}, t\right)=x$, where $x \in \overline{c_{1} c_{2}}$ with $d_{T}\left(c_{1}, x\right)=t$. Note that $\Phi$ is an isometry. We must show that $\Phi: Z \rightarrow \partial X$ is a homeomorphism (same point sets, different topologies). Since $Z$ is compact and $\Phi$ is a bijection, it suffices to show that $\Phi$ is continuous. Let $\left(z_{k}\right) \subset Z$ with $z_{k} \rightarrow z$. Pulling back to the product, we have $z=(a, b, t), z_{k}=\left(a_{k}, b_{k}, t_{k}\right)$, where $a, a_{k} \in C_{1}, b, b_{k} \in C_{2}$ and $t, t_{k} \in[0, \pi / 2]$.

We will show that $\Phi\left(z_{k}\right) \rightarrow \Phi(z)$. For $t=0, \Phi(z)=a$. Consider the sequence $\left(a_{k}\right) \subset C_{1} \subset \partial X$. Since $a_{k} \rightarrow a$ and $d_{T}\left(a_{k}, \Phi\left(z_{k}\right)\right)=t_{k}$ by definition, we have $\Phi\left(z_{k}\right) \rightarrow a=\Phi(z)$ by lower semi-continuity of the Tits metric. The proof is similar if $t=\pi / 2$. If $t \in(0, \pi / 2)$, then $a_{k} \rightarrow a$ and $b_{k} \rightarrow b$ (this is not true in the other two cases). By Theorem 2.4, any cluster point $p$ of $\left(\Phi\left(z_{k}\right)\right)$ lies on the join $\operatorname{arc} \overline{a b}$. By lower semi-continuity, $d_{T}\left(p, C_{1}\right) \leq t$ and $d_{T}\left(p, C_{2}\right) \leq \pi / 2-t$. It follows that $d_{T}\left(p, C_{1}\right)=t$, and so $p=\Phi(z)$, so $\Phi\left(z_{k}\right) \rightarrow \Phi(z)$. Thus $\Phi$ is a homeomorphism and a Tits isometry.

For any two such CAT(0) group boundaries, we get the boundary isomorphism by composing the map $\Phi$ from one with the $\Phi^{-1}$ from the other.

\section{Suspension of the join of two Cantor sets}

We have proven that the join of two Cantor sets $C_{1}$ and $C_{2}$ is Tits rigid. We want to prove that the suspension of it, ie $\Sigma\left(C_{1} * C_{2}\right)$, is also Tits rigid. We first need a result from dimension theory. We will use inductive dimension, which is equivalent to covering dimension in our setting.

We define $\operatorname{dim} \varnothing=-1$. We say that $Z$ has dimension $\leq k$ at a point $z \in Z$ if for any neighborhood $U$ of $z$, there is a neighborhood $V \subset U$ of $z$ with $\operatorname{dim} \partial V \leq k-1$. We say that $Z$ has dimension $\leq k$ if $Z$ has dimension $\leq k$ at each point.

Lemma 3.1 If $Z$ is a compact metrizable space of dimension $k$, then the suspension $\Sigma Z$ of $Z$ has dimension $k+1$.

Proof Since $Z$ is compact and $(0,1)$ is one-dimensional, then by [7, page 34],

$$
\operatorname{dim}[Z \times(0,1)]=\operatorname{dim} Z+\operatorname{dim}(0,1)=k+1 .
$$


So $Z \times(0,1)$ has dimension $\leq k+1$ at each point, with equality in at least one point. Thus $\Sigma Z$ has dimension $\leq k+1$ at each point, with possible exceptions at the suspension points $p$ and $n$.

Every neighborhood $U$ of $p$ will contain a cone neighborhood $V$ of $p$ with $\partial V \cong Z$. Thus the dimension of $\Sigma Z$ at $p$ is at most $\operatorname{dim} Z+1=k+1$, and similarly for $n$. Since $\Sigma Z$ has dimension $\leq k+1$ at each point with equality in at least one point, $\operatorname{dim} \Sigma Z=k+1$.

Definition 3.2 Let $Y$ be a metric space and $A \subset Y$. We say that $A$ is quasidense in $Y$ if, for some $r>0, Y$ is contained in the $r$-neighborhood of $A$.

We now prove a result on the fixed point set of the group action on the boundary $\partial X$.

Lemma 3.3 Let $G$ be a group acting geometrically on a CAT(0) space $X$. If $G$ has a global fixed point $p$, then there is a closed convex quasidense set $\hat{X} \subset X$ with $\hat{X}=\mathbb{R} \times Y$, where $Y$ is a closed convex subset of $\hat{X}$ and $\mathbb{R}$ is an axis of a central element of $G$.

Proof The group $G$ is finitely generated by some elements $g_{1}, \ldots, g_{k}$. By [10, Theorems 3.2 and 3.3], for each $i, p \in \operatorname{Fix}\left(g_{i}\right)=\Lambda \operatorname{Min}\left(g_{i}\right)=\Lambda Z_{g_{i}}$. By [12, Theorem 16],

$$
p \in \bigcap \Lambda Z_{g_{i}}=\Lambda\left[\bigcap Z_{g_{i}}\right]=\Lambda Z_{G},
$$

and, since $Z_{G}$ is convex by [12, Theorem 11], $Z_{G}$ contains an element $g$ of infinite order. By [10, Theorem 3.4], $g$ acts trivially on $\partial X$, so $\partial X=\operatorname{Fix}(g)=\Lambda \operatorname{Min}(g)$. We now let $\hat{X}=\operatorname{Min}(g)$ and apply [2, Chapter II, Theorem 6.8].

Proposition 3.4 Let $X$ be a CAT(0) space and $G$ a group acting geometrically on $X$. The set $A$ of points virtually fixed by $G$ on the boundary is a Tits sphere, and $\partial X=A * Z$ and $\partial_{T} X=A *_{S} Z$, where $Z$ is a compact subset of $\partial X$.

Proof If $A$ is nonempty, then after passing to a subgroup of finite index we may assume that the set of global fixed points of $G$ is nonempty. By Lemma 3.3 there exists a hyperbolic element $h \in Z_{G}$ with endpoints $\{n, p\} \subset A$, and $X$ contains a quasidense subspace which is a product of an axis of $h$ with $X_{1}$, where $X_{1}$ is a closed convex subspace on which $G /\langle h\rangle$ (see [12, Lemma 14]) acts geometrically. Thus $\partial X \cong\{n, p\} * \partial X_{1}=\Sigma \partial X_{1}$, and $\partial_{T} X=\{n, p\} *_{S} \partial_{T} X_{1}=\Sigma_{S} \partial_{T} X_{1}$.

Suppose $\operatorname{dim} \partial X=k(<\infty$ by [12, Theorem 12]). We proceed by induction on $k$. For $k=0$, either $A=\varnothing$, or $\partial X$ is a 0 -sphere, because the 0 -sphere is the only 
0-dimensional space which is a suspension (of the empty set). In the latter case, $A=\partial X$ is a $0-$ sphere.

Assume the result holds for dimension $k-1$. Let $\partial X$ be $k$-dimensional. If $A$ is empty, there is nothing to prove; if not, then $X$ contains a quasidense subset $\mathbb{R} \times X_{1}$, with $\partial X=\{n, p\} * \partial X_{1}$ and $\partial_{T} X=\{n, p\} *_{S} \partial_{T} X_{1}$.

Since $\partial X_{1} \subset \partial X, \partial X_{1}$ is finite-dimensional and by Lemma 3.1, $\operatorname{dim} \partial X_{1}=k-1$. Applying the result to $X_{1}$ with geometric action by $G /\langle h\rangle$, the set $A_{1}$ of all points virtually fixed by $G /\langle h\rangle$ on $\partial X_{1}$ is a Tits sphere. Also $\partial X_{1}=A_{1} * Z_{1}$ and $\partial_{T} X_{1}=$ $A_{1} *_{S} Z_{1}$, where $Z_{1}$ is a compact subset of $\partial X_{1}$. Any point virtually fixed by $G$ in $A-\{n, p\}$ lies on a suspension arc through a unique point $q$ in $\partial X_{1}$. Thus $q$ is virtually fixed by $G$, and also by $G /\langle h\rangle$, so $q \in A_{1}$. It follows that $A$ is the spherical join of $\{n, p\}$ with $A_{1}$, and so $A$ is a Tits sphere in $\partial_{T} X$ and

$$
\partial X=\{n, p\} *\left(A_{1} * Z_{1}\right)=\left[\{n, p\} * A_{1}\right] * Z_{1}=A * Z_{1},
$$

with the same equalities for the spherical joins.

Corollary 3.5 Let $X$ be a CAT(0) space and $G$ a group acting geometrically on $X$. Suppose that $\{n, p\}$ are points on $\partial X$ that are stabilized by all homeomorphisms of $\partial X$. Then the points $n$ and $p$ are the only virtually fixed points of $G$, and there is a closed convex set $Y \subset X$ and $R$ a geodesic line in $X$ satisfying these conditions:

- $R$ is a line from $n$ to $p$.

- There is a closed convex quasidense subset $\hat{X} \subset X$ with $\hat{X}$ decomposing as $Y \times R$.

- $\quad$ The CAT(0) space $Y$ admits a geometric action.

Proof $G$ virtually fixes the points $n$ and $p$, so $n, p \in A$, the sphere of points virtually fixed by $G$, and $\partial X=A * Z$ for some closed subset of $Z$ of $\partial X$. If $A \neq\{n, p\}$, then since any homeomorphism of $A$ with the identity map on $Z$ induces a homeomorphism on their join, there would be homeomorphisms of $\partial X$ that do not stabilize $\{n, p\}$, which contradicts the assumption. So $A=\{n, p\}$ as required. By Lemma 3.3, we get a closed convex quasidense subset $\hat{X} \subset X$, where $\hat{X}=Y \times R$ and $R$ is the axis of a central element $g \in Z_{G}$. Notice that $n$ and $p$ are the endpoints of $R$, since the endpoints will be virtually fixed by $G$ and $n$ and $p$ are the only virtually fixed points. Also, $G /\langle g\rangle$ will act geometrically on $Y$ by [12].

We need the following characterization of an arc.

Theorem 3.6 (Moore [8]) Let $A$ be a compact connected metric space. If $A$ has exactly two noncut points, then $A$ is an arc. 
Lemma 3.7 Let $Y$ be the join of two Cantor sets $C_{1}$ and $C_{2}$. Then the suspension point set $\{n, p\}$ is preserved by homeomorphisms of $\Sigma Y$. If $\Sigma Y$ is also a suspension of a subspace $Z$, then $n$ and $p$ are the $Z$-suspension points as well and $Z$ is isotopic to $Y$ in $\Sigma Y$.

Proof The suspension arcs of $\Sigma Y$ will be called $Y$-suspension arcs. Similarly, we will call the suspension arc of the $Z$-suspension structure $Z$-suspension arcs. We partition $\Sigma Y$ by the local topology:

- The suspension points $\{n, p\}$ which have a neighborhood basis consisting of cone neighborhoods (cones on $Y$ of course), so $\Sigma Y$ is locally connected at $n$ and $p$.

- $\mathcal{C}=\left[\Sigma C_{1} \cup \Sigma C_{2}\right]-\{n, p\}$ (the union of the open $Y$-suspension arcs running through $C_{1}$ and $C_{2}$ ). $\Sigma Y$ is not locally connected at these points. For $p \in \mathcal{C}$ and $U$ a neighborhood of $p$, the component of $U$ containing $p$ is never a 2-manifold (it always contains a topological tri-plane)

- $\mathcal{D}=\Sigma Y-\left[\Sigma C_{1} \cup \Sigma C_{2}\right] . \Sigma Y$ is not locally connected at these points. For $p \in \mathcal{D}$, for $U$ a sufficiently small neighborhood of $p$, the component of $p$ in $U$ will be homeomorphic to an open subset of a disk.

This means that the suspension points of the $Z$-suspension are also $\{n, p\}$, and that this set is fixed by every homeomorphism of $Y$.

Since we can isotope up and down suspension arcs, if $\alpha$ is an open $Z$-suspension arc and $\alpha \cap \mathcal{C} \neq \varnothing$, then $\alpha \subset \mathcal{C}$. (Similarly, if $\alpha \cap \mathcal{D} \neq \varnothing$ then $\alpha \subset \mathcal{D}$.) It follows that, for each $c \in C_{1} \cup C_{2}$, the $Y$-suspension arc through $c$ will be a $Z$-suspension arc as well. Let $c_{1} \in C_{1}$ and $c_{2} \in C_{2}$. Let $\beta \subset C_{1} * C_{2}$ be the join arc from $c_{1}$ to $c_{2}$. The disk $D=\Sigma \beta \subset \Sigma\left(C_{1} * C_{2}\right)=Y$ has boundary $\alpha_{1}$ and $\alpha_{2}$, the ( $Y$ and $\left.Z\right)$-suspension $\operatorname{arcs}$ throughout $c_{1}$ and $c_{2}$, respectively. Let $\omega=Z \cap D$. Since $\alpha_{i}$ is a $Z$-suspension arc, $\alpha_{i} \cap \omega$ is a single point $z_{i}$ (for $i=1,2$ ). We will show that $\omega$ is an arc by showing that $\omega$ is connected and that $z_{1}, z_{2}$ are the only noncut points of $\omega$.

Open $Z$-suspension arcs are disjoint, so $D=\Sigma \omega$. Since $D-\{n, p\} \cong \omega \times I$ (where $I$ is a open interval) is connected, $\omega$ is connected. Notice that $\Sigma\left[\omega-\left\{z_{i}\right\}\right]-\{n, p\} \cong$ [ $\left.D-\alpha_{i}\right]$, which is connected, and so $\omega-\left\{z_{i}\right\}$ is connected. Let $z \in \omega$ with $z \neq z_{i}$ for $i=1,2$. Thus $z \in \operatorname{Int} D$, and so the $Z$-suspension $\operatorname{arc} \gamma$ is contained in $D$, and $\gamma \cap \partial D=\{n, p\}$ since $\gamma$ cannot cross $\alpha_{i}$. It follows that $D-\gamma$ is not connected. Since $D-\gamma \cong[\omega-\{z\}] \times I$, it follows that $\omega-\{z\}$ is not connected. Thus $z$ is a cut point of $\omega$ and by Theorem 3.6, $\omega$ is an arc. 
Notice that $D$ admits a PL structure as a square with vertices $n, c_{1}, p, c_{2}$ and so that the map from $\overline{c_{i} n}$ and $\overline{c_{i} p}$ into $D$ is an isometry, and we can do this in a canonical way for all such $D$. We isotope $\omega$ to the line segment $\hat{\omega}$ in $D$ from $z_{1}$ to $z_{2}$ with the isotopy fixing $\partial D$. We can do this for each such $D$ at the same time. We call the image $Z$ under this isotopy $\hat{Z}$, which is a union of straight line segments in each of our squares.

Now, for each $c \in C_{1} \cup C_{2}$, with $z$ being the unique point of $Z$ on the $Y$-suspension arc $\alpha$ through $c$, we choose an isotopy of $\alpha$ fixing $n$ and $p$ which takes $z$ to $c$. We do these simultaneously and extend linearly on corresponding squares. This gives us an isotopy from $\hat{Z}$ to $Y$ in $\Sigma Y$.

Theorem 3.8 The suspension of the join of two Cantor sets is Tits rigid.

Proof Let $G$ be a group acting geometrically on the CAT(0) space $X$, with $\partial X \cong$ $\Sigma\left[C_{1} * C_{2}\right]$, where $C_{1}$ and $C_{2}$ are Cantor sets. We will show that there is an isometry $\iota: \partial_{T} X \rightarrow \Sigma_{S}\left[C_{1} *_{S} C_{2}\right]$ such that $\iota$ is a homeomorphism from $\partial X$ to $\Sigma\left[C_{1} * C_{2}\right]$.

By Lemma 3.7, every homeomorphism of $\partial X$ fixes the suspension point set $\{n, p\}$. Thus, by Corollary 3.5, there exists a closed convex quasidense subset $\hat{X} \subset X$ with $\hat{X}=Y \times R$, where $Y$ is closed and convex in $X$ and $R$ is a geodesic line from $n$ to $p$. Also, $Y$ admits a geometric action. Now $\partial X=\Sigma \Lambda Y$ and $\partial_{T} X=\Sigma_{S} \Lambda Y$. By Lemma 3.7, $\Lambda Y$ is the join of two Cantor sets. By Lemma 1.8, $\partial Y$ is the join of two Cantor sets. By Theorem 2.7, $\partial_{T} Y$ is the spherical join of two Cantor sets $B_{1}$ and $B_{2}$, where $\partial Y$ is the topological join of $B_{1}$ and $B_{2}$. By Lemma 1.8, in the restriction Tits metric, $\Lambda Y \cong B_{1} *_{S} B_{2}$. Thus $\partial_{T} X$ is isometric to $\Sigma_{S} B_{1} *_{S} B_{2}$, and this gives $\iota$, as required.

\section{Further questions}

We recklessly conjecture that a boundary is Tits rigid if and only if it doesn't have a circle as a join factor. Clearly, the circle is not Tits rigid, and all higher-dimensional spheres will have a circle as a join factor. Thus, every known non-Tits rigid space has a circle as a join factor. Furthermore, if we have a boundary $Z \cong S^{1} * Y$, where $Y$ is also a boundary, we see by taking products that $Z$ will not be Tits rigid.

The first step is to prove that more boundaries are Tits rigid. Possible candidates include the $n$-fold join of Cantor sets and their suspensions or more generally boundaries of CAT(0) cube complexes with certain properties. Visual boundaries of universal covers of Salvetti complexes of right-angled Artin groups may be a source of examples, 
because the known examples can be realized as such. We also imagine that spherical buildings are Tits rigid, but have not examined this at all.

The known Tits rigid boundaries have proper closed invariant subsets, except the Cantor set and the set with two points. This may be a common property for other Tits rigid boundaries. For those that do have closed invariant subsets, is it true that a Tits rigid boundary always has some closed invariant subset which is also Tits rigid with the induced topology?

Also, for a Tits rigid boundary that is not a suspension, is the suspension of this boundary also Tits rigid? Corollary 3.5 is a partial result. The difficulty lies in the fact that there may be nonhomeomorphic topological spaces with homeomorphic joins; an example is given by the double suspension theorem of Cannon and Edwards [3].

In every known Tits rigid boundary, the topology of the Tits boundary resembles the visual topology in the best possible way, ie all the paths in the visual topology are still paths in the Tits boundary. We suspect that this may be the case for other Tits rigid boundaries as well, although this should become much harder to show even for particular cases when the dimension of the visual boundary is at least two.

Recall that a CAT(0) group is rigid if it corresponds to a unique visual boundary up to homeomorphism. There might be nonrigid CAT(0) groups corresponding to some Tits rigid boundaries. Boundaries of known nonrigid CAT( 0$)$ groups are similar in the sense that they are shape equivalent by a result of Bestvina. If one of them is Tits rigid, should every other visual boundary of the same group be Tits rigid too because of their similarity?

\section{References}

[1] W Ballmann, Lectures on spaces of nonpositive curvature, DMV Seminar 25, Birkhäuser, Basel (1995) MR1377265

[2] M R Bridson, A Haefliger, Metric spaces of non-positive curvature, Grundl. Math. Wissen. 319, Springer, Berlin (1999) MR1744486

[3] J W Cannon, Shrinking cell-like decompositions of manifolds: Codimension three, Ann. Math. 110 (1979) 83-112

[4] K L H Chao, CAT(0) spaces with boundary the join of two Cantor sets, Algebr. Geom. Topol. 14 (2014) 1107-1122 MR3180828

[5] C B Croke, B Kleiner, Spaces with nonpositive curvature and their ideal boundaries, Topology 39 (2000) 549-556 MR1746908

[6] D P Guralnik, E L Swenson, A 'transversal' for minimal invariant sets in the boundary of a CAT(0) group, Trans. Amer. Math. Soc. 365 (2013) 3069-3095 MR3034459 
[7] W Hurewicz, H Wallman, Dimension theory, Princeton Mathematical Series 4, Princeton Univ. Press (1941) MR0006493

[8] R L Moore, Concerning the cut-points of continuous curves and of other closed and connected point-sets, Proc. Natl. Acad. Sci. USA 9 (1923) 101-106

[9] P Papasoglu, E Swenson, Boundaries and JSJ decompositions of CAT(0) groups, Geom. Funct. Anal. 19 (2009) 559-590 MR2545250

[10] KE Ruane, Dynamics of the action of a CAT(0) group on the boundary, Geom. Dedicata 84 (2001) 81-99 MR1825346

[11] K Ruane, CAT(0) groups with specified boundary, Algebr. Geom. Topol. 6 (2006) 633-649 MR2220692

[12] E L Swenson, A cut point theorem for CAT(0) groups, J. Differential Geom. 53 (1999) 327-358 MR1802725

Mathematical Sciences Center, Tsinghua University

Beijing 100084, China

Mathematics Department, Brigham Young University

Provo, UT 84602, USA

khek@math.tsinghua.edu.cn, eric@math.byu.edu

Received: 13 March 2014 Revised: 17 July 2014 